\title{
Sex-specific neural activity when resolving cognitive interference in individuals with or without prior internalizing disorders
}

\author{
Zhishun Wang ${ }^{\mathrm{a}, 1}$, Rachel H. Jacobs ${ }^{\mathrm{b}, 1}$, Rachel Marsh ${ }^{\mathrm{c}}$, Guillermo Horga ${ }^{\mathrm{a}}$, Jianping Qiao ${ }^{\mathrm{d}}$, \\ Virginia Warner ${ }^{\mathrm{e}}$, Myrna M. Weissman ${ }^{\mathrm{e}}$, Bradley S. Peterson ${ }^{\mathrm{f}, *}$ \\ a Division of Translational Neuroimaging in the Department of Psychiatry, The New York State Psychiatric Institute and the College of Physicians E Surgeons, \\ Columbia University, New York, NY, USA \\ ${ }^{\mathrm{b}}$ University of Illinois at Chicago, Department of Psychiatry and Institute for Juvenile Research, 1747W, Roosevelt Road M/C 747, Chicago, IL 60608, USA \\ ${ }^{\mathrm{c}}$ Division of Child and Adolescent Psychiatry in the Department of Psychiatry, The New York State Psychiatric Institute and The College of Physicians \& \\ Surgeons, Columbia University, 1051 Riverside Drive, Unit 74, New York, NY 10032, USA \\ e Division of Epidemiology in the Department of Psychiatry, the New York State Psychiatric Institute and the College of Physicians E Surgeons, Columbia \\ University, 1051 Riverside Drive Unit 24, New York, NY 10032, USA \\ d College of Physics and Electronics, Shandong Normal University, Jinan, China \\ ${ }_{\mathrm{f}}^{\mathrm{f}}$ Institute for the Developing Mind, Children's Hospital Los Angeles, Keck School of Medicine at the University of Southern California, 4650 Sunset Blvd. \\ MS\#135, Los Angeles, CA 90027, USA
}

\section{A R T I C L E I N F O}

\section{Article history:}

Received 7 April 2014

Received in revised form

8 June 2015

Accepted 7 July 2015

Available online 21 October 2015

\section{Keywords:}

Internalizing disorders

Sex differences

fMRI

Cognitive interference

Default-mode networks

Anti-correlated networks

\begin{abstract}
A B S T R A C T
The processing of cognitive interference is a self-regulatory capacity that is impaired in persons with internalizing disorders. This investigation was to assess sex differences in the neural correlates of cognitive interference in individuals with and without an illness history of an internalizing disorder. We compared functional magnetic resonance imaging blood-oxygenation-level-dependent responses in both males $(n=63)$ and females $(n=80)$ with and without this illness history during performance of the Simon task. Females deactivated superior frontal gyrus, inferior parietal lobe, and posterior cingulate cortex to a greater extent than males. Females with a prior history of internalizing disorder also deactivated these regions more compared to males with that history, and they additionally demonstrated greater activation of right inferior frontal gyrus. These group differences were represented in a significant sex-by-illness interaction in these regions. These deactivated regions compose a task-negative or default mode network, whereas the inferior frontal gyrus usually activates when performing an attention-demanding task and is a key component of a task-positive network. Our findings suggest that a prior history of internalizing disorders disproportionately influences functioning of the default mode network and is associated with an accompanying activation of the task-positive network in females during the resolution of cognitive interference.
\end{abstract}

(c) 2016 Published by Elsevier Ireland Ltd.

\section{Introduction}

Internalizing disorders, including depression and anxiety, are characterized by a tendency to turn negative emotions inward when experiencing distress, as opposed to turning them outward, which is observed in externalizing disorders. The fact that females

\footnotetext{
* Corresponding author

E-mail addresses: wangz@nyspi.columbia.edu (Z. Wang), rjacobs@psych.uic.edu (R.H. Jacobs), marshr@nyspi.columbia.edu (R. Marsh), horgag@nyspi.columbia.edu (G. Horga), qiaojianpingdsp@gmail.com (J. Qiao), warnerv@nyspi.columbia.edu (V. Warner), weissman@nyspi.columbia.edu (M.M. Weissman), bpeterson@chla.usc.edu (B.S. Peterson).

${ }^{1}$ The authors contributed equally.
}

are at greater risk for internalizing disorders than males is well established (Eaton et al., 2012). Internalizing disorders, such as depression and anxiety, are highly comorbid and derive from common mechanisms, such as neuroticism (Hettema et al., 2006). Moreover, a large body of work examines the phenotypic presentation of internalized distress across development (ZahnWaxler et al., 2000). Continuity between depression and anxiety, particularly among adolescent girls, has been observed (Costello et al., 2003); yet limited work examines sex differences in the neural underpinnings of internalizing illness.

We used a self-regulatory control task to examine whether sexrelated differences may emerge among males and females who have a history of an internalizing disorder. Deficits in the self-regulatory control that is required to resolve cognitive interference have been 
implicated in the etiology of internalizing disorders, both in experimental (Austin et al., 2001; Joormann and Gotlib, 2008; Joormann et al., 2010) and neuroimaging (Berman et al., 2011; Pizzagalli, 2011) studies. To date few neuroimaging studies (Keller and Menon, 2009; Weissman-Fogel et al., 2010) have assessed sex differences in the functioning of neural circuits underlying cognitive or attentional processes. Such studies have the potential to explicate sex-specific mechanisms that may contribute to the documented higher prevalence of internalizing disorders among females (Angold and Worthman, 1993; Nolen-Hoeksema et al., 1999; Cyranowski et al., 2000; Kuehner, 2003). The current study assesses sex differences in the neural processing of cognitive interference and examines whether a previous history of an internalizing disorder is associated with different patterns of brain activations in males and females.

Self-regulatory control is required to resolve cognitive interference. Tasks such as the Stroop and Simon require activation of prefrontal regions to ignore a prepotent stimulus feature and respond in a task-relevant manner. Parallel, anti-correlated neural networks function in concert to support these self-regulatory processes. Fronto-striatal and fronto-parietal regions form a taskpositive network that routinely increases in activity during task performance (Fox et al., 2005). Task-negative regions typically deactivate when individuals engage in goal-directed behavior (Shulman et al., 1997). These latter regions, including the posterior cingulate cortex (PCC), precuneus (PCu) (Uddin et al., 2009), inferior parietal lobe (IPL) and superior frontal gyrus (SFG), have collectively been labeled the Default Mode Network (DMN) (De Luca et al., 2006; Buckner et al., 2008). Relative deactivation of the DMN during a goal-directed task compared to baseline or a relatively easier condition is hypothesized to represent either greater activity while engaging in autobiographical or self-referential mind-wandering during the easier condition or greater active suppression of activity during an active or more challenging condition (i.e., responding to a condition requiring greater attention such as in the case of incongruent stimuli). Indeed, deactivation in medial parietal and medial frontal regions is hypothesized to reflect interruptions of internal introspection in the service of external attention-demanding tasks (Gusnard and Raichle, 2001; Hayden et al., 2009; Peterson et al., 2009; Peterson et al., 2014). Individuals with depression have demonstrated reduced cortical involvement in the resolution of interference when assessed with the Stroop task (Chechko et al., 2013), but sex differences during the resolution of cognitive interference, particularly as they relate to a history of internalizing disorders, have not been examined.

The current study is an exploratory examination of sex differences in brain activation during the performance of the Simon task in both the presence and absence of a history of an internalizing disorder. We examined internalizing disorders by examining both depression and anxiety diagnoses based on prior studies within the same cohort showing both illnesses to occur at elevated rates among high risk individuals and that an anxiety disorder often precedes the development of depression (Warner et al., 2008). Detecting significant sexby-illness interactions would suggest that the effects of prior illness on brain systems that support the resolution of cognitive interference differ between females and males. We hypothesized that females would demonstrate longer reaction times than males and would deactivate DMN regions and activate task-positive regions to a greater extent than males during the resolution of cognitive interference; in addition, females with a history of an internalizing disorder would demonstrate the greatest deactivations.

\section{Methods}

We obtained functional MRI (fMRI) scans in 143 individuals, ages 7-54 years, who belonged to a 3-generation cohort followed through 5 waves of clinical assessments over more than 20 years, thereby ensuring an excellent, prospectively acquired knowledge of the psychiatric history of all participants. Diagnostic interviews across all waves (Weissman et al., 2005) were conducted using a semistructured diagnostic instrument (the Schedule for Affective Disorders and Schizophrenia-Lifetime Version for adults, and a child version of the instrument that was modified for DSM-IV (American Psychological Association, 1994)) for participants 6-17 years of age (Mannuzza et al., 1986). The original project design was to follow offspring of the original Generation 1 cohort to examine high and low familial risk for Major Depressive Disorder (MDD). This study was initiated in 1982. Both the high and low risk groups were Generations 2 and 3 offspring of Generation 1. Generation 1 individuals were recruited from an outpatient clinic and were being treated for moderate to severe MDD with functional impairment and the offspring of these individuals formed the high risk group. In addition, the Generations 2 and 3 offspring of additional Generation 1 adults with no history of psychiatric illness recruited from the same community formed the low risk group. Wave 5 fMRI scans occurred between 2002 and 2007. This current sample consists of offspring (2nd and 3rd generation) of the original cohort who were consented to completing an fMRI scan. High and low risk was the original design of the study, but due to the low frequency of high risk females who did not develop illness, we specifically compare offspring with a previous diagnosis of Major Depressive Disorder or anxiety disorder, including Generalized Anxiety Disorder and Social Phobia and who were therefore categorized as previously "ill". Past history as well as current mental illness was assessed using the structured diagnostic interviews described above. Data from those with a history of illness were compared to those without a history of illness.

We assessed the main effects of sex in addition to sex-by-illness effects on brain activity. fMRI scan data were acquired from 143 individuals (40 children younger than 18, 103 adults). Scans were attempted but unsuccessful in 20 individuals. For technical reasons, fMRI scans were not attempted in another 8 individuals. Complete methods regarding this longitudinal study are described elsewhere (Weissman et al., 2005; Weissman et al., 2006; Peterson et al., 2014). The Children's Depression Rating Scale-Revised (CDRSR) for youth and the Hamilton Depression Rating Scale (HAM-D) for adults were converted into a $z$-score for each participant to index depression severity. The Revised Children's Manifest Anxiety Scale (RCMAS) and the Hamilton Anxiety Rating Scale (HAM-A) were also converted to a $z$-score to index anxiety severity.

\subsection{Stimulus presentation}

Visual stimuli were presented through MRI-compatible goggles (Resonance Technologies, Inc.). A series of white arrows pointing either left or right were displayed against a black background either to the left or right of a white gaze fixation cross-hair positioned at midline. The majority of stimuli were "congruent" arrows pointing in the same direction as their position on the screen (e.g. a rightward-pointing arrow presented to the right of midline). A smaller number of stimuli ( $7 \%$ ) were "incongruent", pointing in a direction opposite their position on the screen (e.g. a left-pointing arrow presented to the right of midline), spaced pseudorandomly every 13-16 congruent stimuli. Participants were instructed to respond as quickly as possible to the direction of the arrow by pressing a button on a response box. Stimulus duration was $1300 \mathrm{~ms}$, with an inter-stimulus interval of $350 \mathrm{~ms}$. Each run was composed of 102 stimuli (2.97 min duration), and each participant performed 10 runs. All stimuli were presented with E-Prime software 1.1 (Psychology Software Tools, Inc., Sharpsburg, PA 15215, USA) running on a Pentium-IV PC. A schematic of the Simon task is presented in Fig. 1.

\subsection{Behavioral data analysis}

Reaction times (RTs) and accuracy scores on each trial of the Simon task were entered as dependent variables in separate repeated measures, linear mixed models in SAS (SAS Institute Inc, Carey, NC) with risk group (high, low), illness (previously ill, healthy), stimulus congruence (incongruent, congruent), age, sex, and run number $(0-10)$ included as independent variables. The effect of sex and prior illness on performance (RTs or accuracy) of congruent and incongruent trials was assessed by the statistical significance of the sex-by-illness interaction. We used an ANCOVA to assess differences across previously ill and healthy groups in interference scores, calculated as the difference in mean RTs during correct performance on the incongruent and congruent stimulus trials.

\subsection{Image acquisition}

Images were acquired on a Siemens Sonata $1.5 \mathrm{~T}$ scanner (Siemens AG, Munich, Germany) using a standard quadrature head coil. In all participants, a 3D spoiled gradient recall image was acquired for co-registration with the axial functional images and with the MNI (Montreal Neurological Institute) coordinate system. Functional images were acquired using a single shot gradient echo planar pulse sequence in groups of 16 axial slices per volume and 102 volumes per run. We acquired 10 runs for each participant. Parameters for the echoplanar images were $\mathrm{TR}=1650 \mathrm{msec}, \mathrm{TE}=30 \mathrm{~ms}$, flip angle $=90^{\circ}$, acquisition matrix $=64 \times 64$, 


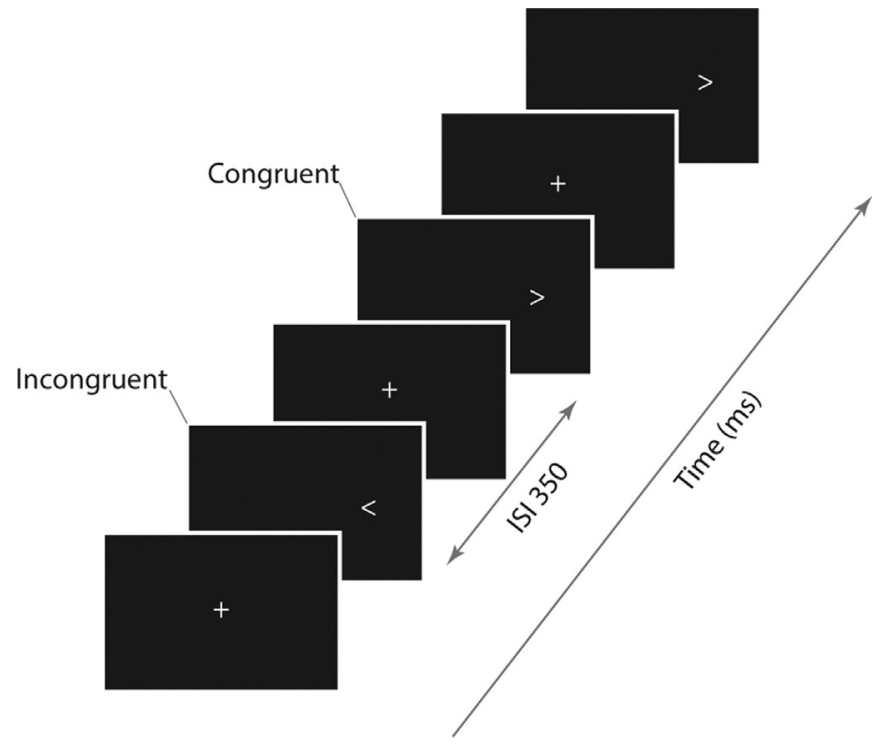

Fig. 1. Simon task.

field of view $=20 \mathrm{~cm} \times 20 \mathrm{~cm}$, slice thickness $=8 \mathrm{~mm}$, skip $=0.5 \mathrm{~mm}$, receiver bandwidth $=62.5 \mathrm{kHz}$, in-plane resolution $=3.125 \mathrm{~mm} \times 3.125 \mathrm{~mm}$. Each run lasted $2.97 \mathrm{~min}$, for a total EPI scan time of $29.70 \mathrm{~min}$.

\subsection{Image preprocessing}

We ran a preprocessing software package in batch mode under MATLAB (Mathworks, Natick, MA, USA) that was developed in-house by integrating processing functions from SPM (http://www.fil.ion.ucl.ac.uk/spm/) and FSL (http:// www.fmrib.ox.ac.uk/fsl/) for the preprocessing of functional images. Images with a motion greater than one voxel were excluded for all the subsequent analyses. Motion-corrected images of each participant were co-registered to the corresponding T1-weighted high-resolution anatomical image, which in turn was spatially normalized to an MNI template space with isotropic voxel dimensions of $3 \times 3 \times 3 \mathrm{~mm}^{3}$.

\subsection{Image analysis}

A first-level analysis used a General Linear Model with a weighted leastsquares algorithm to model the fMRI time series for each participant. A secondlevel analysis detected functional activity associated with the task within and between groups using a Bayesian-based group analysis (Neumann and Lohmann, 2003; Klein et al., 2007). All analyses were performed using SPM8 (http://www.fil. ion.ucl.ac.uk/spm/) operating under MATLAB 2009B.

\subsubsection{First-level}

In each design matrix, except the constant, we had 6 independent regressors that represented the conditions of fixation, congruent correct (the participant performed the congruent trials correctly), incongruent correct (the participant performed the incongruent trials correctly), congruent incorrect (the participant performed the congruent trials incorrectly), incongruent incorrect (the participant performed the incongruent trials incorrectly), and missing response (the participants did not respond). The t-contrast of incongruent correct vs. congruent correct (interference effect) was then used in the second-level analyses to detect task-related activity within and between the groups (sex, diagnosis and their interaction).

\subsubsection{Second-level}

We used a Bayesian inference approach (Neumann and Lohmann, 2003; Klein et al., 2007) to detect random effects of group differences from the first-level outcomes by assessing the posterior probability of detecting a within- or betweengroup difference, $\beta$, given the activation map attained in a particular contrast. We used a posterior probability $>99.99 \%$ as the threshold for statistical significance in each of the contrast maps. Conventional second-level analysis strategies employ parametric inference to detect group effects in a statistical parametric map by rejecting the null hypothesis $(\beta=0)$ at each voxel of the image. In contrast, a group effect using a Bayesian approach infers the posterior probability of detecting the observed group effect $(\beta \neq 0)$ given the data in a Posterior Probability Map (Neumann and Lohmann, 2003). Whereas voxel-wise tests require correction for the number of comparisons performed, the Bayesian method, which infers posterior probability, does not generate false positives and does not require adjustment of $p$ - values. Numerous simulations and empirical studies confirm these features of Bayesian analyses (Friston et al., 2002; Friston and Penny, 2003).

To examine the main effect of sex, we compared interference maps (the contrast of Incongruent Correct vs. Congruent Correct or IC vs. CC) between all male participants and all female participants, controlling for risk group, history of prior illness, age, and interference scores. To assess sex-specific illness effects, we tested the significance of the sex-by-illness interaction of IC vs. CC contrast maps included in this model. We present separate interference maps comparing males and females with a history of illness and maps comparing males and females who have never been ill while controlling for risk, age, and interference scores. All results reported reflect the accurate resolution of cognitive interference and represent the contrast of incongruent correct versus congruent correct.

\section{Results}

\subsection{Participants}

Participant characteristics are detailed in Table 1. More females $(56 / 83,67 \%)$ had diagnoses of depression or anxiety than males (27/83, 33\%; $\left.\chi^{2}=10.66, p<0.01\right)$. Individuals without a history of illness were significantly younger $(M=24.9, \mathrm{SD}=14.1)$ than previously ill individuals $(M=33.7, \mathrm{SD}=11.6)$ at the time of the Wave $5 \operatorname{scan}(t=-4.07, p<0.01)$. Eleven individuals met current criteria for MDD or anxiety at the time of the scan, and 25 were taking psychotropic medication. We control for these confounding variables by conducting post-hoc analyses as detailed below.

\subsection{Behavioral performance}

We compared the reaction times (RTs) for task performance during congruent trials, during incongruent trials, and the average of the two RTs between ill females and ill males, as well as between healthy females and healthy males. The three RTs were all greater in ill females than in ill males $(509.99 \pm 9.70$ vs. $466.37 \pm 13.85, p<0.02$ for RT congruent; $697.44 \pm 10.89$ vs. $625.73 \pm 16.20, p<0.0004$ for RT incongruent; $603.72 \pm 9.71$ vs. $546.05 \pm 14.44, p<0.002$ for RT average). There were no significant differences between healthy females and healthy males (Fig. 2 A-C). We performed similar analyses on the accuracy measure of task performance during congruent trials and during incongruent trials. No significant differences in accuracy across sexes or illness groups were observed. We also analyzed the correlations between these reaction times and the continuous measure (Z-scores) of symptom severity scores of depression in the females and the males, respectively. Illness symptoms were significantly correlated with reaction times during congruent trials among females $(r=0.304, p<0.03)$ but not in males $(r=0.048$, $p>0.8$ ) (Fig. 2 D). Furthermore, we also analyzed the correlations between these reaction times and the ages of the subjects, but we did not find any significant correlations between age and performance on the task.

\subsection{Sex differences in brain activation}

Sex differences were detected as main effects in numerous cortical regions, including posterior cingulate (PCC; BA 31), posterior temporal/inferior parietal lobe (IPL; BA 39), and superior frontal gyri (SFG; BA 10; Fig. 3), deriving from greater deactivation of these regions in females than in males during correct responses on incongruent compared to congruent trials. Sex differences were also detected in small portions of frontoparietal regions, including bilateral inferior parietal cortex (IPC; BA 40) and right insula, and derived from greater activation in females compared to males. Peak coordinates are presented in Table 2. 


\subsection{Sex-by-illness interactions}

We previously identified illness effects in DMN regions including the PCC (Peterson et al., 2014). Controlling for familial risk, disproportionally greater deactivation of DMN regions in previously ill females compared to previously ill males illustrated that the sex differences documented in this network were exaggerated among those with a history of depression or anxiety (Fig. 4; Table 2). Within the previously ill but not healthy group, greater deactivation of the left IPL (BA 39) was observed in females compared to males during correct responses on incongruent trials compared to correct responses on congruent trials. Within the previously ill group, greater activation of the right IFG (BA 9) and deactivation of the right SFG (BA 8) and bilateral PCu (BA 7) was detected in females compared to males. Fig. 5 illustrates extracted beta weights for each of the four groups in the SFG. Overall, previously ill females most strongly deactivated DMN regions and activated attention regions when accurately resolving interference.

\subsection{Potential confounds}

Findings were similar when excluding the 25 participants ( 9 males, 16 females) taking a psychotropic medication at the time of the scan (11 of whom met current criteria for MDD or anxiety). When including only individuals who had passed through the window of risk (aged 25 or older) the significant age difference between groups was eliminated $(t=0.89, p=0.37)$ and results did not change (Supplementary Figs. 1 and 2 and Supplementary Table 1).

\section{Discussion}

We examined the independent and interactive effects of sex and previous internalizing illness (depression and/or anxiety) on neural activity when resolving cognitive interference during the Simon task. The main effect of sex indicates that although both sexes activated cortical areas required for the allocation of attention and cognitive control, and although both sexes deactivated DMN regions during correct responses to incongruent stimuli compared to correct responses to congruent stimuli, females deactivated portions of the DMN (left IPL, bilateral SFG and PCC) to a greater extent than males when accurately resolving this

Table 1

Demographic and clinical characteristics.

\begin{tabular}{|c|c|c|c|c|c|}
\hline \multirow[t]{2}{*}{ Characteristic } & \multirow{2}{*}{$\begin{array}{l}\text { No history of } \\
\text { illness (HC) } \\
N=60\end{array}$} & \multirow{2}{*}{$\begin{array}{l}\text { History of illness } \\
N=83\end{array}$} & \multicolumn{3}{|c|}{ Group comparison } \\
\hline & & & Test statistic & df & $p$-value \\
\hline Age & $24.9 \pm 14.1$ & $33.7 \pm 11.6$ & $t=-4.01$ & 141 & $<0.01$ \\
\hline Sex & $\mathrm{F}=24, \mathrm{M}=36$ & $\mathrm{~F}=56, \mathrm{M}=27$ & $\chi^{2}=10.7$ & 1 & $<0.01$ \\
\hline $\begin{array}{l}\text { Recruitment } \\
\text { group }\end{array}$ & $\begin{array}{l}\mathrm{LR}=38 \\
\mathrm{HR}=22\end{array}$ & $\mathrm{LR}=22, \mathrm{HR}=61$ & $\chi^{2}=19.4$ & 1 & $<0.01$ \\
\hline Lifetime MDD & $N=0$ & $\begin{array}{l}N=60 \text { (23 MDD } \\
\text { only } 37 \\
\text { MDD + anxiety) }\end{array}$ & $\chi 2=19.3$ & 1 & $<0.01$ \\
\hline $\begin{array}{l}\text { Lifetime anxi- } \\
\text { ety disorder }\end{array}$ & $N=0$ & $\begin{array}{l}N=60 \text { ( } 23 \text { Anxi- } \\
\text { ety only } 37 \\
\text { MDD + anxiety) }\end{array}$ & $\chi^{2}=74.7$ & 1 & $<0.01$ \\
\hline $\begin{array}{l}Z \text { anxiety } \\
\text { score }\end{array}$ & $-0.30 \pm 0.79$ & $0.19 \pm 1.07$ & $t=-3.30$ & 124 & $<0.002$ \\
\hline $\begin{array}{l}Z \text { depression } \\
\text { score }\end{array}$ & $-0.35 \pm 0.66$ & $0.23 \pm 1.11$ & $t=-2.69$ & 123 & $<0.01$ \\
\hline
\end{tabular}

Note. $\mathrm{F}=$ female, $\mathrm{M}=$ male, $\mathrm{LR}=$ low risk, $\mathrm{HR}=$ high risk, $\mathrm{MDD}=$ major depressive disorder, $\mathrm{HC}=$ health controls. The sample consisted of $40 \mathrm{~h}$ ill females, $21 \mathrm{~h}$ ill males, 6 LR HC females, 16 LR HC males, 16 LR ill females, 6 LR ill males, 18 LR HC females, and 20 LR HC males. interference. The neural pattern associated with previous illness (reported previously; (Peterson et al., 2014)) was also associated with greater deactivations of DMN regions. Sex-by-illness interactions presented a unique pattern of neural activity in which task-negative DMN regions (IPL, SFG, and PCu) deactivated to a greater extent in ill females compared to ill males. In addition, a task-positive region, the IFG, simultaneously activated more among ill females compared to ill males. Thus, sex-by-illness interactions indicate that previous illness influences functioning of the DMN, as well as a specific task-positive hyperactivity in the IFG in females to a greater extent than in males. On the other hand, the analyses of task performance behavioral data suggested that ill females responded more slowly than ill males regardless of task stimulus type (congruent or incongruent trials). Moreover, decreased reaction times among ill females correlated with illness symptom severity scores, indicating that the illness had particularly influenced females to perform the task and thus they had to work harder than males to complete it. This explains why we detected hyperactivity in a portion of the task-positive network in ill females (with a history of internalizing disorder).

These robust sex differences in neural circuits supporting the resolution of cognitive interference are notable due to the relative dearth of fMRI research examining sex differences in brain activity. Reports of performance differences on cognitive interference tasks have been inconsistent, but some evidence suggests that males and females may engage different cognitive strategies to resolve cognitive interference (Mekarski et al., 1996). Findings from fMRI studies probing cognitive processes other than interference resolution offer some support for sex-specific patterns of brain activity in the DMN. For example, a study using a mental arithmetic task found greater activations in DMN regions among males compared to females in regions typically deactivated during complex cognitive tasks (Keller and Menon, 2009). Other data indicate increased prefrontal activations in females and increased parietal activations in males when performing a visual-spatial oddball task (Bell et al., 2006; Christakou et al., 2009), suggesting that males and females may use different strategies when completing cognitive tasks. Some resting state functional connectivity studies report sex differences (Biswal et al., 2010), such as greater functional connectivity between DMN regions in females compared to males (Zuo et al., 2010), while others were either limited by single sex samples (Kunisato et al., 2011) or fail to detect sex differences (Weissman-Fogel et al., 2010). Further research is required to explicate sex differences in cognitive functioning and in the resolution of cognitive interference.

Alternatively, females may require greater suppression of internally directed attention or free-associative thought during the more challenging incongruent condition. Attention to a cognitive task requires reallocating processing resources toward external stimuli, producing task-induced deactivation of internal mental activities (McKiernan et al., 2003). Activation in the anterior cingulate, PCC, and STG has been positively associated with response speed to stimuli detected in unpredictable locations (Hahn et al., 2007) and the PCC has been hypothesized to provide resources for automatic allocation of attention towards unpredictable targets that may be important for survival (Gusnard et al., 2001).

From a theoretical perspective, we were interested in identifying possible sex differences in the DMN because of its association with rumination (Cooney et al., 2010) and depression (Sheline et al., 2010), in addition to recent research indicating greater connectivity within the DMN network in females compared to males (Zuo et al., 2010). Rumination is a perseverative thought pattern representing a maladaptive attempt to regulate mood. Women are more likely than men to ruminate when sad or dysphoric (Nolen-Hoeksema et al., 1999), and evidence suggests that rumination may contribute to the development of depression 


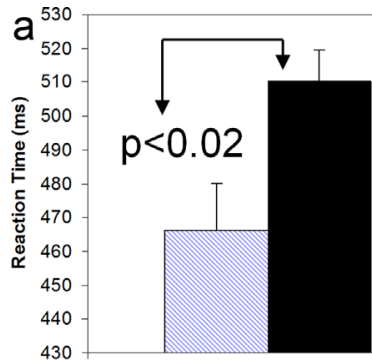

III

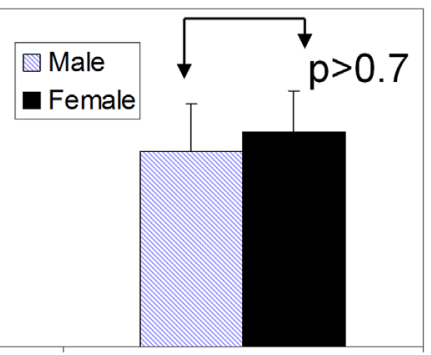

Healthy

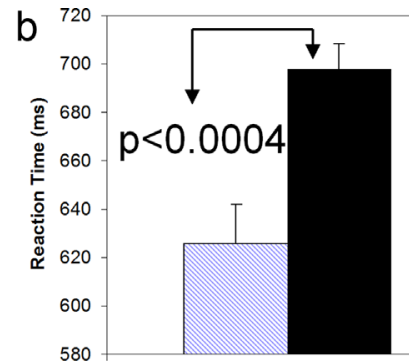

III

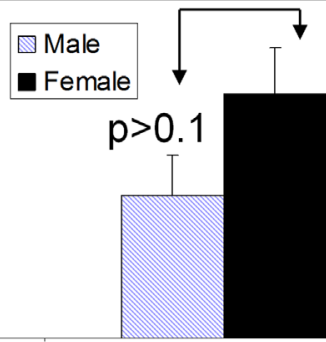

Healthy

C
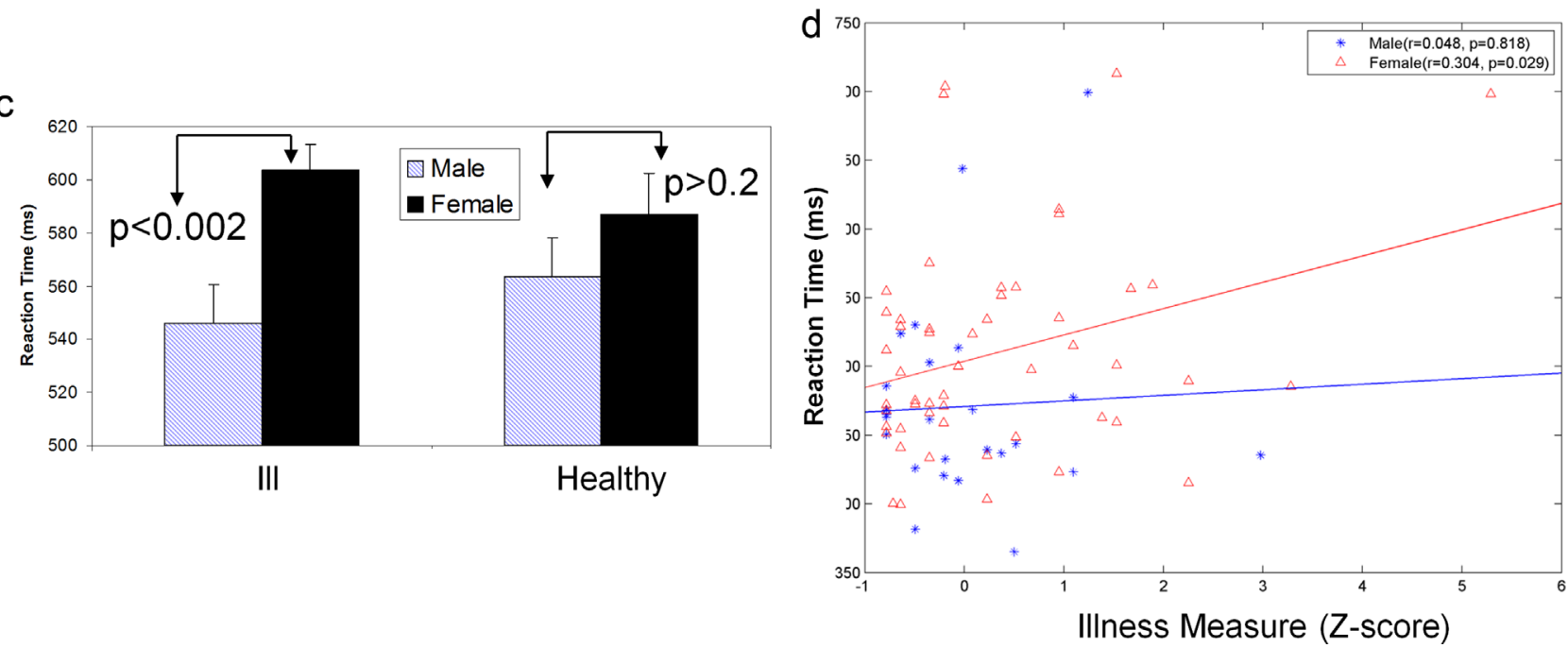

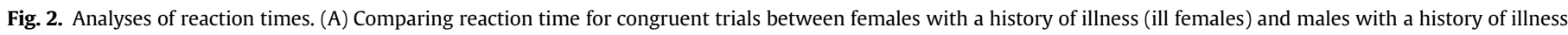

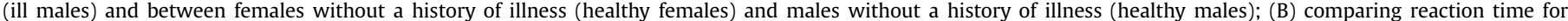

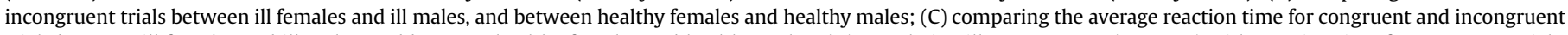

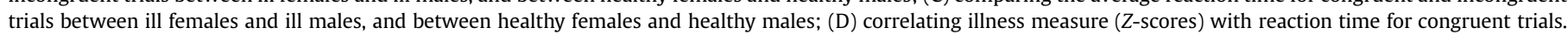

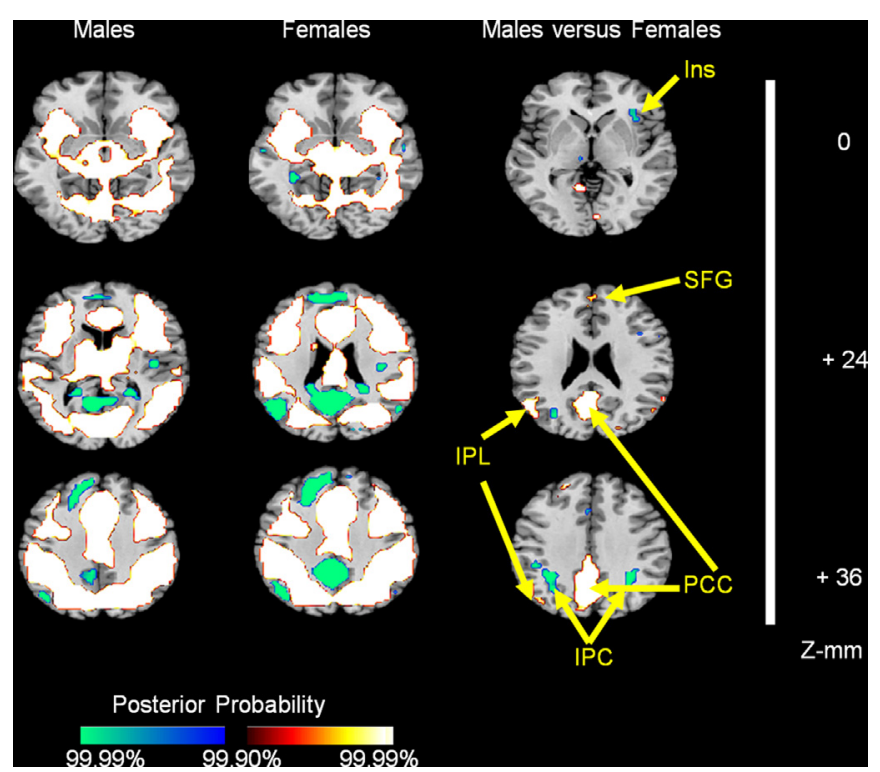

Fig. 3. Sex differences in brain activation during interference. Posterior probability thresholds $>99.90 \%$ in axial slices showing group average brain activation maps (posterior means) of interference effects (incongruent vs. congruent stimulus contrasts) in males and females. Sex differences were detected in frontoparietal and default mode regions in male and female participants. Increases in signal during correct incongruent trials relative to correct congruent trials are shown in red, and decreases are shown in blue. Ins, insula; IPC, inferior parietal cortex; IPL, inferior parietal lobe; PCC, posterior cingulate cortex; SFG, superior frontal gyrus. (For interpretation of the references to color in this figure legend, the reader is referred to the web version of this article.)
Table 2

Peak coordinates for the contrast of incongruent correct versus congruent correct across all subjects.

\begin{tabular}{|c|c|c|c|c|c|c|c|}
\hline \multirow[t]{2}{*}{ Region } & \multicolumn{2}{|c|}{ Location/BA } & \multirow[t]{2}{*}{ No. voxels } & \multicolumn{3}{|c|}{ Peak location } & \multirow[t]{2}{*}{$Z$ score } \\
\hline & & & & $x$ & $y$ & $z$ & \\
\hline \multicolumn{8}{|l|}{ Main effect of sex } \\
\hline $\begin{array}{l}\text { Posterior cingulate } \\
\text { cortex }\end{array}$ & $\mathrm{R} / \mathrm{L}$ & 31 & 1175 & 0 & -36 & 48 & 7.14 \\
\hline \multirow{2}{*}{$\begin{array}{l}\text { Inferior parietal } \\
\text { cortex }\end{array}$} & $\mathrm{R}$ & 40 & 142 & 33 & -51 & 39 & -5.19 \\
\hline & $\mathrm{L}$ & 40 & 170 & -33 & -48 & 36 & -4.60 \\
\hline Inferior parietal lobe & $\mathrm{L}$ & 39 & 129 & -51 & -60 & 30 & 7.11 \\
\hline $\begin{array}{l}\text { Superior frontal } \\
\text { gyrus }\end{array}$ & $\mathrm{R}$ & 10 & 91 & 3 & 54 & 6 & 4.65 \\
\hline Insula & $\mathrm{R}$ & 13 & 30 & 36 & 24 & 0 & -3.54 \\
\hline \multicolumn{8}{|c|}{ Sex-by-illness interaction } \\
\hline Precuneus & $\mathrm{R}$ & 7 & 118 & 6 & -72 & 36 & 5.39 \\
\hline $\begin{array}{l}\text { Superior frontal } \\
\text { gyrus }\end{array}$ & $\mathrm{R}$ & 8 & 34 & 24 & 36 & 39 & 4.18 \\
\hline $\begin{array}{l}\text { Inferior parietal } \\
\text { cortex }\end{array}$ & $\mathrm{L}$ & 19 & 48 & -39 & -72 & 39 & -4.69 \\
\hline Inferior frontal gyrus & $\mathrm{R}$ & 9 & 30 & 54 & 6 & 30 & -4.50 \\
\hline
\end{tabular}

(Nolen-Hoeksema et al., 2008). Rumination impairs self-regulatory control by promoting perseveration and reducing cognitive flexibility (Davis and Nolen-Hoeksema, 2000), which further impairs the resolution of cognitive interference (Joormann et al., 2010). Sex differences in rumination, in addition to the higher prevalence rates of depression among females compared to males (Angold and Worthman, 1993; Nolen-Hoeksema et al., 1999; Cyranowski et al., 2000; Kuehner, 2003), offer an interesting interpretation of 


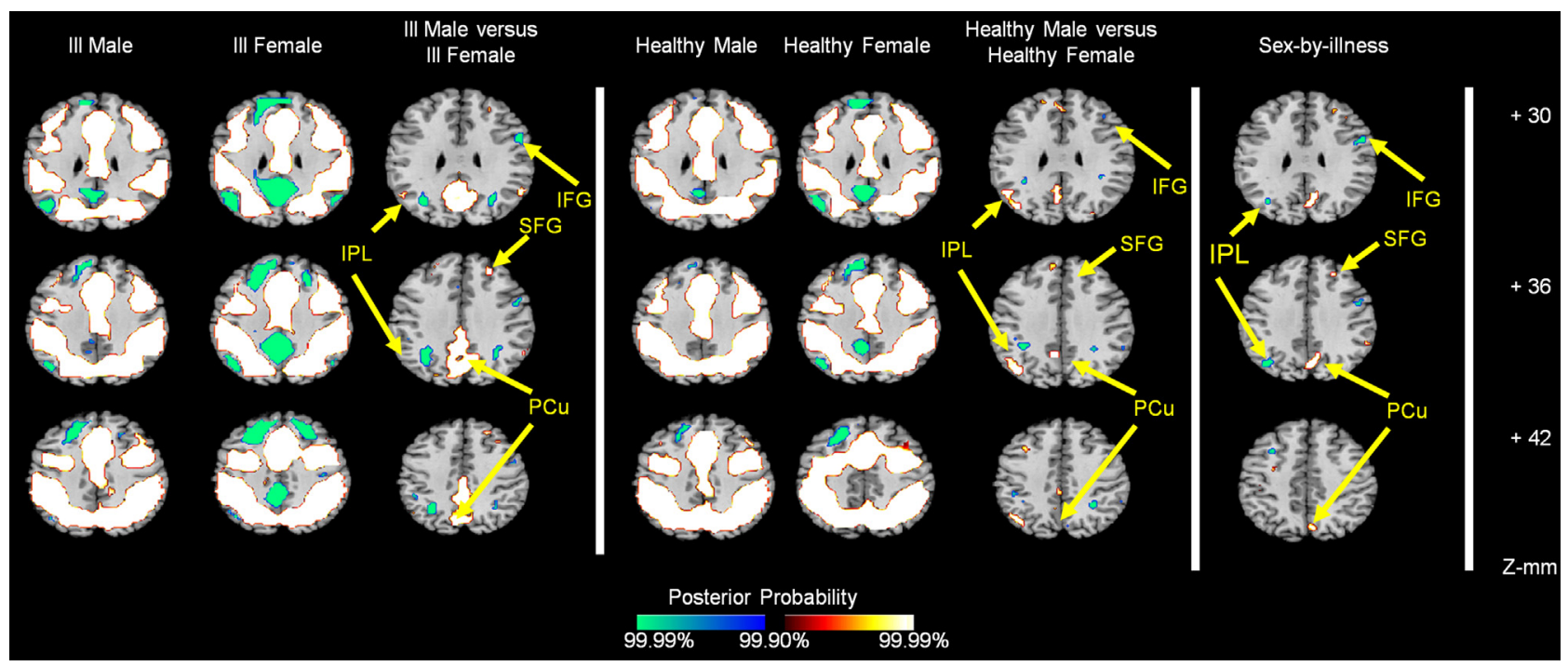

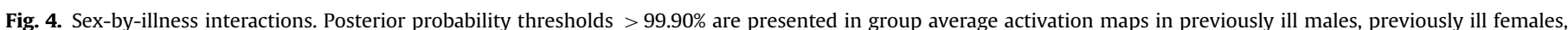

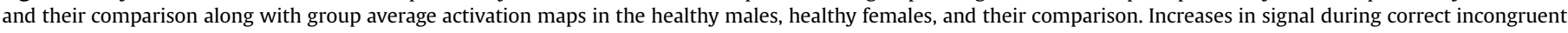

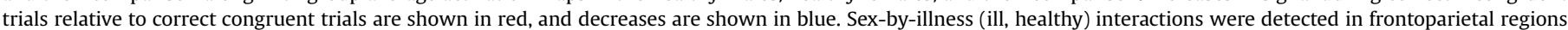

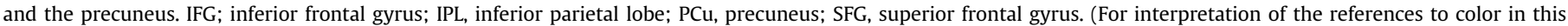
figure legend, the reader is referred to the web version of this article.)

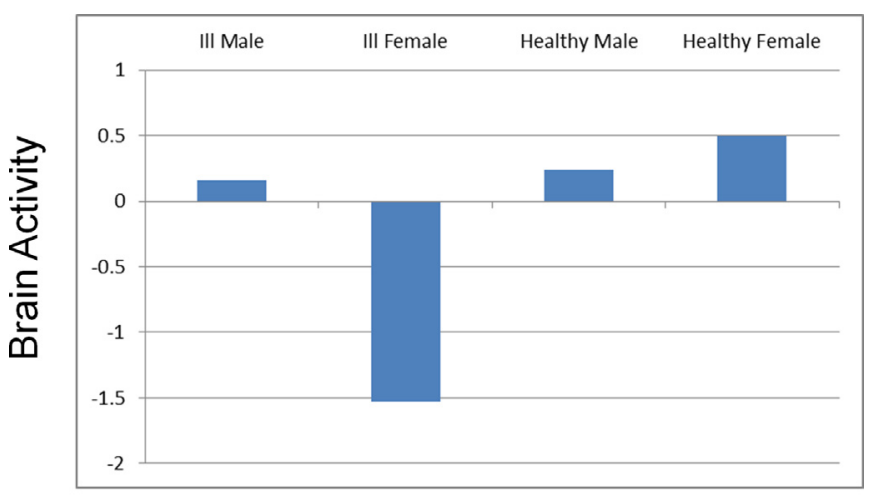

Sex by Diagnosis Group

Fig. 5. BOLD response in the superior frontal gyrus among males and females with and without a history of depression and anxiety.

the current results. One possible explanation for our finding of greater deactivations in DMN regions during cognitive interference may stem from greater levels of free-associative thought during the relatively easier congruent condition of the Simon task, consistent with the role of the DMN in mind-wandering (Mason et al., 2007). Among individuals with depression, DMN activity may dominate task-related activity, and this increased DMN dominance may be associated with increased levels of rumination (Hamilton et al., 2011). Future research can directly test whether sex-related differences in DMN regions are related to the construct of rumination using tasks that induce rumination in the scanner as well as examine the DMN "at rest". We believe our results highlight the importance of examining sex and sex interactions particularly when studying internalizing disorders and our exploratory findings can direct future work designed to explicitly test these hypotheses.

Cortical inefficiency among females with current MDD has been observed using the Stroop task (Wagner et al., 2006), but that study did not include males for comparison. Greater activation in the rostral anterior cingulate and dorsolateral prefrontal cortex was observed among females with active illness compared to female healthy controls. In contrast, we did not find a main effect of sex in these regions; however, it is possible that this is due either to our stringent threshold requiring posterior probability greater than $99.90 \%$ or to differences between the Stroop and the Simon tasks. Notably, the Stroop uses verbal stimuli and the Simon does not, and therefore our results do not recruit regions of the brain contributing to the processing of verbal stimuli, perhaps explaining some of the differences.

Our study has several limitations. First, although well powered, our sample was heterogeneous, as we chose to conduct an exploratory analysis of internalizing illness for the purpose of generating hypotheses for future research. Second, we also had too few children to specifically examine the effects of sex and sex-byillness among youth separately, and therefore the pattern of results we present may not apply to youth. It will be important for future research to examine the development of networks including the DMN in both females and males as they pass through the adolescent window of risk. It is also likely that there is a burden effect of multiple episodes of illness that may drive the current findings, which may not be present among youth. Future research can test whether the number of episodes or comorbidities, both reflecting either an increased burden of illness or "treatment refractoriness", are associated with greater deactivations of the DMN. In addition, we did not collect resting-state functional connectivity data and therefore cannot determine whether differences in DMN functioning are related to increased connectivity during the resting state between nodes of this network. Several studies have documented increased connectivity within the DMN among acute, chronic, and remitted MDD populations (Greicius et al., 2007; Sheline et al., 2010; Posner et al., 2013). Future studies should additionally consider inducing rumination or directly measuring mind-wandering to better understand how DMN activations and deactivations function within each sex group and how this may interact with illness course to reduce the ability to resolve cognitive interference. Our findings should also be replicated with alternative tasks of cognitive interference such as the Stroop. Lastly, future research could examine how menstrual and hormonal cycles influence brain functioning and how sex may interact with illness in predicting sex-specific brain activation patterns during the resolution of cognitive interference.

Our findings indicate the critical importance of examining sex differences in the neural correlates of cognitive capacities influenced 
by internalizing disorders, and they add to a growing literature implicating aberrant DMN functioning in mental illness (Buckner et al., 2008; Broyd et al., 2009; Hamilton et al., 2012; Marchetti et al., 2012). We suggest that female sex as well as a history of an internalizing disorder contributes to deactivations of the task-negative DMN and increased activity in the task-positive network, presenting an increased anti-correlation between the two networks during the resolution of cognitive interference. Recent evidence indicates that DMN activity can be modulated with Selective Serotonin Reuptake Inhibitors (SSRIs;(McCabe et al., 2011)) and that antidepressants restore normal patterns of function in the DMN (Delaveau et al., 2011; Posner et al., 2013). This evidence highlights the importance of understanding the functioning of the DMN in internalizing disorders for the purposes of advancing intervention research and designing novel treatments directly targeting this dysfunction. Perhaps such treatments would be more effective in attenuating illness in females, given their greater tendency towards exaggerated deactivations of the DMN.

\section{Acknowledgments}

Funded by NIMH Grants 5R01MH036197 (PIs Weissman \& Peterson), 5P50MH090966 (PI Gingrich), and 5T32MH016434 (PI Peterson), and R01MH090062 (PI Marsh). The authors would like to thank Dr. Scott Langenecker for his feedback on this manuscript.

Disclosures: Drs. Wang, Jacobs, Marsh, Horga, Qiao, Warner, and Peterson have none. In the past three years, Dr. Weissman received funding from the National Institute of Mental Health (NIMH), the National Institute on Drug Abuse (NIDA), the National Alliance for Research on Schizophrenia and Depression (NARSAD), the Sackler Foundation, the Templeton Foundation and receives royalties from the Oxford University Press, Perseus Press, the American Psychiatric Association Press, and MultiHealth Systems.

\section{Appendix A. Supplementary material}

Supplementary data associated with this article can be found in the online version at http://dx.doi.org/10.1016/j.pscychresns.2015.07.008.

\section{References}

Angold, A., Worthman, C.W., 1993. Puberty onset of gender differences in rates of depression: a developmental, epidemiologic and neuroendocrine perspective. J. Affect. Disord. 29, 145-158.

American Psychological Association, 1994. Diagnostic and Statistical Manual of Mental Disorders, 4th ed. American Psychiatric Association, Washington.

Austin, M.P., Mitchell, P., Goodwin, G.M., 2001. Cognitive deficits in depression possible implications for functional neuropathology. Br. J. Psychiatry 178, 200-206.

Bell, E.C., Willson, M.C., Wilman, A.H., Dave, S., Silverstone, P.H., 2006. Males and females differ in brain activation during cognitive tasks. Neuroimage 30 , 529-538.

Berman, M.G., Nee, D.E., Casement, M., Kim, H.S., Deldin, P., Kross, E., Gonzalez, R., Demiralp, E., Gotlib, I.H., Hamilton, P., Joormann, J., Waugh, C., Jonides, J., 2011. Neural and behavioral effects of interference resolution in depression and rumination. Cogn. Affect. Behav. Neurosci. 11, 85-96.

Biswal, B.B., Mennes, M., Zuo, X.N., Gohel, S., Kelly, C., Smith, S.M., Beckmann, C.F., Adelstein, J.S., Buckner, R.L., Colcombe, S., Dogonowski, A.M., Ernst, M., Fair, D., Hampson, M., Hoptman, M.J., Hyde, J.S., Kiviniemi, V.J., Kotter, R., Li, S.J., Lin, C.P., Lowe, M.J., Mackay, C., Madden, D.J., Madsen, K.H., Margulies, D.S., Mayberg, H. S., McMahon, K., Monk, C.S., Mostofsky, S.H., Nagel, B.J., Pekar, J.J., Peltier, S.J., Petersen, S.E., Riedl, V., Rombouts, S.A., Rypma, B., Schlaggar, B.L., Schmidt, S., Seidler, R.D., Siegle, G.J., Sorg, C., Teng, G.J., Veijola, J., Villringer, A., Walter, M., Wang, L., Weng, X.C., Whitfield-Gabrieli, S., Williamson, P., Windischberger, C., Zang, Y.F., Zhang, H.Y., Castellanos, F.X., Milham, M.P., 2010. Toward discovery science of human brain function. Proc. Natl. Acad. Sci. U. S. A. 107, 4734-4739.

Broyd, S.J., Demanuele, C., Debener, S., Helps, S.K., James, C.J., Sonuga-Barke, E.J., 2009. Default-mode brain dysfunction in mental disorders: a systematic review. Neurosci. Biobehav. Rev. 33, 279-296.
Buckner, R.L., Andrews-Hanna, J.R., Schacter, D.L., 2008. The brain's default network: anatomy, function, and relevance to disease. Ann. N. Y. Acad. Sci. 1124, $1-38$.

Chechko, N., Augustin, M., Zvyagintsev, M., Schneider, F., Habel, U., Kellermann, T. 2013. Brain circuitries involved in emotional interference task in major depression disorder. J. Affect. Disord. 149, 136-145.

Christakou, A., Halari, R., Smith, A.B., Ifkovits, E., Brammer, M., Rubia, K., 2009. Sexdependent age modulation of frontostriatal and temporo-parietal activation during cognitive control. Neuroimage 48, 223-236.

Cooney, R.E., Joormann, J., Eugene, F., Dennis, E.L., Gotlib, I.H., 2010. Neural correlates of rumination in depression. Cogn. Affect. Behav. Neurosci. 10, 470-478.

Costello, E.J., Mustillo, S., Erkanli, A., Keeler, G., Angold, A., 2003. Prevalence and development of psychiatric disorders in childhood and adolescence. Arch. Gen. Psychiatry 60, 837-844.

Cyranowski, J.M., Frank, E., Young, E., Shear, M.K., 2000. Adolescent onset of the gender difference in lifetime rates of major depression: a theoretical model. Arch. Gen. Psychiatry 57, 21-27.

Davis, R.N., Nolen-Hoeksema, S., 2000. Cognitive inflexibility among ruminators and nonruminators. Cogn. Ther. Res. 24, 699-711.

De Luca, M., Beckmann, C.F., De Stefano, N., Matthews, P.M., Smith, S.M., 2006. fMRI resting state networks define distinct modes of long-distance interactions in the human brain. Neuroimage 29, 1359-1367.

Delaveau, P., Jabourian, M., Lemogne, C., Guionnet, S., Bergouignan, L., Fossati, P., 2011. Brain effects of antidepressants in major depression: a meta-analysis of emotional processing studies. J. Affect. Disord. 130, 66-74.

Eaton, N.R., Keyes, K.M., Krueger, R.F., Balsis, S., Skodol, A.E., Markon, K.E., Grant, B. F., Hasin, D.S., 2012. An invariant dimensional liability model of gender differences in mental disorder prevalence: evidence from a national sample. J. Abnorm. Psychol. 121, 282-288.

Fox, M.D., Snyder, A.Z., Vincent, J.L., Corbetta, M., Van Essen, D.C., Raichle, M.E., 2005. The human brain is intrinsically organized into dynamic, anticorrelated functional networks. Proc. Natl. Acad. Sci. U. S. A. 102, 9673-9678.

Friston, K.J., Penny, W., 2003. Posterior probability maps and SPMs. Neuroimage 19 1240-1249.

Friston, K.J., Penny, W., Phillips, C., Kiebel, S., Hinton, G., Ashburner, J., 2002. Classical and Bayesian inference in neuroimaging: theory. Neuroimage 16, 465-483.

Greicius, M.D., Flores, B.H., Menon, V., Glover, G.H., Solvason, H.B., Kenna, H., Reiss, A.L., Schatzberg, A.F., 2007. Resting-state functional connectivity in major depression: abnormally increased contributions from subgenual cingulate cortex and thalamus. Biol. Psychiatry 62, 429-437.

Gusnard, D.A., Raichle, M.E., 2001. Searching for a baseline: functional imaging and the resting human brain. Nat. Rev. Neurosci. 2, 685-694.

Gusnard, D.A., Raichle, M.E., Raichle, M.E., 2001. Searching for a baseline: functional imaging and the resting human brain. Nat. Rev. Neurosci. 2, 685-694.

Hahn, B., Ross, T.J., Stein, E.A., 2007. Cingulate activation increases dynamically with response speed under stimulus unpredictability. Cereb. Cortex 17, 1664-1671.

Hamilton, J.P., Etkin, A., Furman, D.J., Lemus, M.G., Johnson, R.F., Gotlib, I.H., 2012. Functional neuroimaging of major depressive disorder: a meta-analysis and new integration of base line activation and neural response data. Am. J. Psychiatry $169,693-703$.

Hamilton, J.P., Furman, D.J., Chang, C., Thomason, M.E., Dennis, E., Gotlib, I.H., 2011. Default-mode and task-positive network activity in major depressive disorder: implications for adaptive and maladaptive rumination. Biol. Psychiatry 70, 327-333.

Hayden, B.Y., Smith, D.V., Platt, M.L., 2009. Electrophysiological correlates of default-mode processing in macaque posterior cingulate cortex. Proc. Natl. Acad. Sci. 106, 5948-5953.

Hettema, J.M., Neale, M.C., Myers, J.M., Prescott, C.A., Kendler, K.S., 2006. A population-based twin study of the relationship between neuroticism and internalizing disorders. Am. J. Psychiatry 163, 857-864.

Joormann, J., Gotlib, I.H., 2008. Updating the contents of working memory in depression: interference from irrelevant negative material. J. Abnorm. Psychol. 117, 182-192.

Joormann, J., Nee, D.E., Berman, M.G., Jonides, J., Gotlib, I.H., 2010. Interference resolution in major depression. Cogn. Affect. Behav. Neurosci. 10, 21-33.

Keller, K., Menon, V., 2009. Gender differences in the functional and structural neuroanatomy of mathematical cognition. Neuroimage 47, 342-352.

Klein, T.A., Neumann, J., Reuter, M., Hennig, J., von Cramon, D.Y., Ullsperger, M., 2007. Genetically determined differences in learning from errors. Science 318 $1642-1645$

Kuehner, C., 2003. Gender differences in unipolar depression: an update of epidemiological findings and possible explanations. Acta Psychiatr. Scand. 108, 163-174.

Kunisato, Y., Okamoto, Y., Okada, G., Aoyama, S., Demoto, Y., Munakata, A., Nomura M., Onoda, K., Yamawaki, S., 2011. Modulation of default-mode network activity by acute tryptophan depletion is associated with mood change: a resting state functional magnetic resonance imaging study. Neurosci. Res. 69, 129-134.

Mannuzza, S., Fyer, A.J., Klein, D.F., Endicott, J., 1986. Schedule for affective disorders and schizophrenia - lifetime version modified for the study of anxiety disorders (SADS-LA): rationale and conceptual development. J. Psychiatr. Res. 20, 317-325.

Marchetti, I., Koster, E.H., Sonuga-Barke, E.J., De Raedt, R., 2012. The default mode network and recurrent depression: a neurobiological model of cognitive risk factors. Neuropsychol. Rev. 22, 229-251.

Mason, M.F., Norton, M.I., Van Horn, J.D., Wegner, D.M., Grafton, S.T., Macrae, C.N., 
2007. Wandering minds: the default network and stimulus-independent thought. Science 315, 393-395.

McCabe, C., Mishor, Z., Filippini, N., Cowen, P.J., Taylor, M.J., Harmer, C.J., 2011. SSRI administration reduces resting state functional connectivity in dorso-medial prefrontal cortex. Mol. Psychiatry 16, 592-594.

McKiernan, K.A., Kaufman, J.N., Kucera-Thompson, J., Binder, J.R., 2003. A parametric manipulation of factors affecting task-induced deactivation in functional neuroimaging. J. Cogn. Neurosci. 15, 394-408.

Mekarski, J.E., Cutmore, T.R., Suboski, W., 1996. Gender differences during processing of the Stroop task. Percept. Motor Skill 83, 563-568.

Neumann, J., Lohmann, G., 2003. Bayesian second-level analysis of functional magnetic resonance images. Neuroimage 20, 1346-1355.

Nolen-Hoeksema, S., Larson, J., Grayson, C., 1999. Explaining the gender difference in depressive symptoms. J. Personal. Soc. Psychol. 77, 1061-1072.

Nolen-Hoeksema, S., Wisco, B.E., Lyubomirsky, S., 2008. Rethinking Rumination. Perspect. Psychol. Sci. 3, 400-424.

Peterson, B.S., Potenza, M.N., Wang, Z., Zhu, H., Martin, A., Marsh, R., Plessen, K.J., Yu, S., 2009. An FMRI study of the effects of psychostimulants on default-mode processing during Stroop task performance in youths with ADHD. Am. J. Psychiatry 166, 1286-1294.

Peterson, B.S., Wang, Z., Horga, G., Warner, V., Rutherford, B., Klahr, K.W., Graniello B., Wickramaratne, P., Garcia, F., Yu, S., Hao, X., Adams, P.B., Qian, M., Liu, J., Gerber, A., Weissman, M.M., 2014. Discriminating risk and resilience endophenotypes from lifetime illness effects in familial major depressive disorder. J. Am. Med. Assoc. Psychiatry 71, 136-148.

Pizzagalli, D.A., 2011. Frontocingulate dysfunction in depression: toward biomarkers of treatment response. Neuropsychopharmacology 36, 183-206.

Posner, J., Hellerstein, D.J., Gat, I., Mechling, A., Klahr, K., Wang, Z., McGrath, P.J., Stewart, J.W., Peterson, B.S., 2013. Antidepressants normalize the default mode network in patients with dysthymia. J. Am. Med. Assoc. Psychiatry 70, 373-382.

Sheline, Y.I., Price, J.L., Yan, Z., Mintun, M.A., 2010. Resting-state functional MRI in depression unmasks increased connectivity between networks via the dorsal nexus. Proc. Natl. Acad. Sci. U. S. A. 107, 11020-11025.

Shulman, G.L., Fiez, J.A., Corbetta, M., Buckner, R.L., Miezin, F.M., Raichle, M.E., Petersen, S.E., 1997. Common blood flow changes across visual tasks.2. Decreases in cerebral cortex. J. Cogn. Neurosci. 9, 648-663.

Uddin, L.Q., Kelly, A.M., Biswal, B.B., Castellanos, F.X., Milham, M.P., 2009. Functional connectivity of default mode network components: correlation, anticorrelation, and causality. Hum. Brain Mapp. 30, 625-637.

Wagner, G., Sinsel, E., Sobanski, T., Kohler, S., Marinou, V., Mentzel, H.J., Sauer, H., Schlosser, R.G., 2006. Cortical inefficiency in patients with unipolar depression: an event-related FMRI study with the Stroop task. Biol. Psychiatry 59, 958-965.

Warner, V., Wickramaratne, P., Weissman, M.M., 2008. The role of fear and anxiety in the familial risk for major depression: a three-generation study. Psychol. Med. 38, 1543-1556.

Weissman, M.M., Wickramaratne, P., Nomura, Y., Warner, V., Pilowsky, D., Verdeli, H., 2006. Offspring of depressed parents: 20 years later. Am. J. Psychiatry 163, $1001-1008$

Weissman, M.M., Wickramaratne, P., Nomura, Y., Warner, V., Verdeli, H., Pilowsky, D.J. Grillon, C., Bruder, G., 2005. Families at high and low risk for depression: a 3-generation study. Arch. Gen. Psychiatry 62, 29-36.

Weissman-Fogel, I., Moayedi, M., Taylor, K.S., Pope, G., Davis, K.D., 2010. Cognitive and default-mode resting state networks: do male and female brains "rest" differently? Hum. Brain Mapp. 31, 1713-1726.

Zahn-Waxler, C., Klimes-Dougan, B., Slattery, M.J., 2000. Internalizing problems of childhood and adolescence: prospects, pitfalls, and progress in understanding the development of anxiety and depression. Dev. Psychopathol. 12, 443-466.

Zuo, X.N., Kelly, C., Di Martino, A., Mennes, M., Margulies, D.S., Bangaru, S. Grzadzinski, R., Evans, A.C., Zang, Y.F., Castellanos, F.X., Milham, M.P., 2010. Growing together and growing apart: regional and sex differences in the lifespan developmental trajectories of functional homotopy. J. Neurosci. 30, 15034-15043. 